\title{
Multiple muscle contractures in left lower limb in an adult
}

\begin{abstract}
Introduction: Muscle contractures may be rarely seen in adults as continuation of congenital deformity, malformations or post infective/ ischemic fibrosis of muscles. Putting a normal muscle in an abnormal non- functioning state for some length of time causes shortening of muscle leading to contracture and sub sequent joint deformity.

Case report: A 40 years adult presenting with contractures of left lower limb hip, knee, ankle and toes following inadequately treated soft tissue infection in calf and heel two years back has been treated by skeletal traction, followed with release of tight fascia at hip, thigh and tenotomy of medial hamstring tendons resulting in partial improvement of contractures. He is planned for hamstring release, posterior capsulotomy of knee, tendoachilles lengthening, bracing and mobilization after soft tissue healing in functional position, in a gradual manner. The case is being reported due to rare occurrence of acquired multiple contractures in adulthood and to highlight the importance of position of joints in any painful condition.
\end{abstract}

Message: Deep seated soft tissue infection can present late as contractures if proper positioning and antigravity mobilization of muscles is not done regularly after suitable treatment of infection. Shortened length of muscles due to any reason may lead to contractures and joint deformities.
Volume 3 Issue 6 - 2018

\author{
Naresh Kumar S Dhaniwala,' Mukund \\ Naresh Dhaniwala ${ }^{2}$ \\ 'Department of orthopedics, Datta Meghe Institute of Medical \\ Sciences, India \\ ${ }^{2}$ Department of orthopedics, Rajawadi Hospital, india
}

\begin{abstract}
Correspondence: Naresh Kumar S Dhaniwala, Department of orthopedics, JNMC \& AVBRH, Datta Meghe Institute of Medical Sciences, Sawangi (Meghe), Wardha 44200I, India, Email nsdhanewala@gmail.com
\end{abstract} Received: November 18, 2018 | Published: November 30,
2018

\section{Introduction}

Deformity is defined in English dictionary as 'the state of being deformed or imperfection/ disfigurement in a particular part of body'. It may occur due to fracture, infection, arthritis, tumor or congenital deficiency or malformation. Lower limb deformities in children occur commonly due to congenital deficiencies in limb formation or due to complication resulting from infection of bone or joint. In deformities or growth disturbance occurring in children there are many methods of measurement and prediction of resulting deficiency. ${ }^{1}$ Deformity is defined medically as a congenital or acquired angular and/or rotational formation of a bone or a malalignment of bones at a joint, such as congenital dislocation of hip, coxa vara, genu valgus etc. Malformation is considered as 'made wrong', not just crooked, rotated or malaligned, such as hemimelia, polydactyly, synostosis etc. ${ }^{2}$ A muscle contracture on the other hand, is a permanent adaptive shortening of a muscle which occurs when a normally innervated muscle is held immobile in a shortened position for some period of time. ${ }^{3}$ Contractures can cause deformities in response to prolonged spasticity or abnormal posture in which muscle remains in a shortened state. In adults deformities may be due to untreated congenital conditions, infective pathology or malunion of fractures. In India persons having suffered from poliomyelitis, may be seen with various deformities due to muscle contractures resultant from muscle imbalance in the limb. In general, adults present with deformities rarely. Herein, a case of multiple contractures leading to deformities in left lower limb hip, knee, ankle and toes is being reported due to its rarity and uncertain etiology.

\section{Case report}

A 40 years old male presented in orthopedic service of a tertiary medical care center in the central India, with the chief complaints of deformity in left lower limb with inability in normal ambulation for two years. The patient was asymptomatic two years back, when he developed a small swelling each in left upper calf region and at left heel. The swellings were painful and caused burning sensation in the limb. The swelling at the heel burst on its own and discharged a little amount of purulent fluid. The patient then started walking on toes of the left foot, keeping the knee in bent position. He found relief in lying in lateral position with left side down and kept his left hip, knee and ankle in flexed position. Though, treated at the local health center, the heel wound took six months to heal, by which time the patient kept his left lower limb joints in flexion most of the time. Then, he noticed that he is unable to straighten his left hip, knee, ankle and toes normally and cannot walk normally. Not getting any relief by local doctor's treatment he was referred to our center in one of the multi diagnostic camps organized in his area. The patient did not have any relevant past medical history. He was never hospitalized. He was married, had two children and never suffered from any sexually transmitted disease or fever before, during and after development of deformities.

On general examination, the patient was thin built, with normal higher functions. Systemic examination was normal except a small non- tender, freely mobile granuloma in his right breast. He was walking with the help of two crutches and right lower limb, keeping his left lower limb hanging in air due to deformities. Examination of musculo-skeletal system revealed normal spine, normal upper limbs and normal right lower limb. On the left lower limb, he had 30 degrees fixed flexion deformity (further movement from 30 to 90 degree) and 10 degrees abduction deformity (further abduction from 10 to 30 ) at left hip, with restricted rotations which could not be assessed due to abnormal position of the limb. The left knee had 110 degrees flexion deformity with further flexion up to 160 degrees. The left ankle was in 30 degrees fixed plantar flexion deformity and all the toes also had plantar flexion deformity. Sensation in the limb was normal and power normal in the muscles. There were three firm, mildly tender, granulomatous swellings of size $2 \mathrm{~cm} . x 4 \mathrm{~cm}$., in subcutaneous location, one each in left lower thigh, left upper calf and at the left 
heel. Adductors of left hip, iliotibial tract, hamstring muscles and gastro soleus muscles were found tight and contracted. X- ray of the knee (Figure 1) and pelvis did not reveal any gross bony abnormality except the flexed position, though proper x-rays could not be done due to extreme flexion at knee. CT scan or MRI of knee also could not be done due to the abnormal positioning of the limb. The patient did not agree initially for FNAC (Fine Needle Aspiration Cytology) from the swellings. All blood parameters were found within normal limits, including tests for HIV after counselling.

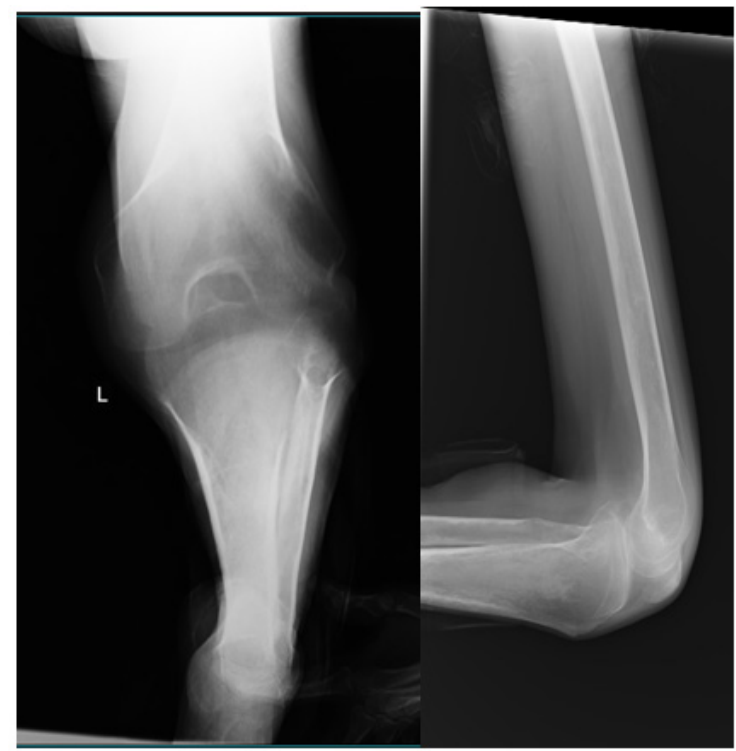

Figure I Anteroposterior and lateral view of left knee. Ap view shows foreshortened leg due to extreme deformity. Lateral view shows 100 degrees flexion deformity at knee without any bony or soft tissue abnormality.

In view of the required aim of correction of the deformities, the patient was put on lower tibial pin traction using Denham pin. The hip could be brought to neutral rotation and on further examination rotations were found within normal range, excluding any hip pathology. Knee flexion deformity gradually decreased to 90 degree. After 10 days of skeletal traction, the patient was submitted to soft tissue release under anesthesia. This included deep fascia release at Tensor fascia latae muscle at outer \& anterior ilium (Soutter's release), iliotibial tract release by multiple incisions in lower thigh (Yount's release), tenotomy of tight medial hamstring tendons namely semitendinosus, semimembranosus and release of tight plantar fascia $\&$ ligaments in the sole of left foot. On table correction of knee could be achieved up to 50 degrees under anesthesia. The patient was further continued on traction but the patient avoided traction due to pain, so further deformity could be not be corrected (Figure 2). The patient is now planned for further surgery including medial \& lateral gastrocnemius muscles release from its origin at lower femur \& posterior capsulotomy of the left knee to achieve full correction of the knee deformity and tendoachilles lengthening to correct plantar flexion deformity of ankle. This will be followed by plaster slab/ cast application for 4-6 weeks along with muscle strengthening and mobilizing exercises. The lateral hamstring, Biceps femoris will be kept intact to help in the knee flexion. The patient will require measures in the form of the night splints for 6 months and active mobilization of knee \& ankle to prevent recurrence of the deformity.

The granulomatous swelling at left heel is reported as keratized skin. FNAC of calf swelling is reported as organized hematoma on cytology and ultrasonography examination. The patient continues under treatment.

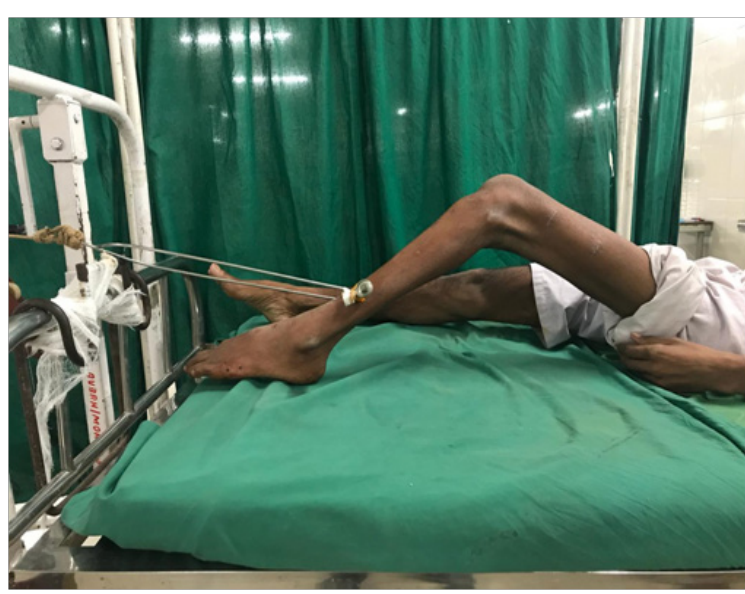

Figure $\mathbf{2}$ shows the patient with lower tibial traction with flexion of hip, knee and ankle.

\section{Discussion}

Acquired Deformities in adults are rare except when due to congenital, neuromuscular or post infective causes. Contractures develop when normal elastic tissues are replaced by inelastic tissue. This results in shortening and hardening of tissues, leading to joint deformities and loss of movement of the joint. Traction and adaptive yoga have been noted to reduce contractures. ${ }^{4}$

Contractures cause disability due to reduced motor performance, mobility limitations, loss of functions for activities of daily living and increasing pain on attempted motion. Multifactorial in etiology, myopathic conditions cause more severe contracture than neuropathic causes. ${ }^{5}$ Known extrinsic causes for contractures include inability to actively move a joint through its full range of motion, static positioning for prolonged period, and agonist antagonist muscle imbalance. ${ }^{6}$ Intrinsic factors are fibrotic changes in the muscle leading to decreased extensibility. ${ }^{7}$ Contractures in overlying skin such as post burn or post- surgery, contractures in subcutaneous fascia such as Dupuytren's contracture can also cause joint deformity. ${ }^{8}$ Muscle contracture due to deep seated infection or fibrosis of muscle due to ischemia or contracture owing to muscle imbalance as seen in cerebral palsy, post- polio residual palsy, tendon rupture are common. ${ }^{8}$ Lower limb contractures are more common than upper limb contractures.

The described case did not have any history supporting the possibility of trauma or acute infection. He had infection in his left heel which was not treated properly and he developed plantar flexion (equinus) deformity in ankle and toes. Improper positioning of knee and hip and lack of active use and exercises of limb further caused flexion deformity at knee and hip. Infection probably spread to the calf muscles also and created a picture like Volkmann ischemic contracture due to its deep-seated location. This aggravated ankle and knee contracture along with causing flexion deformities in toes also. This is supported by the clinical examination.

Inability to move actively through the full range is the single most frequent factor contributing to the occurrence of fixed contractures. Less than antigravity knee extension puts a person at risk for knee flexion contractures, more so if the patient does not ambulate and keeps his knee flexed most of the time. A shortened muscle length may cause up to a $40 \%$ loss of sarcomeres. ${ }^{5}$ The static nature of wheelchair mobilization in comparison to the dynamic movement with gait may causes limb contracture.

Bracing, stretching, traction and various types of surgical 
intervention such as tendon lengthening, tendon release, tendon transfer, muscle slide surgery, capsulotomy, osteotomy, arthrodesis are utilized in the prevention and treatment of contractures as per the need in a case. ${ }^{9}$ Combination of methods may be needed to manage cases of contractures. In mild contractures, active exercises, corrective splint and passive stretching may suffice. In lower limbs, traction is quite useful. It can be coupled with active mobilization to gain strength in the muscles. Fasciotomy, release of tight fascial structures, muscles and maintenance splints with exercises are usually sufficient for moderate contractures. In severe contracture, pre- surgery traction followed by suitable surgery in single or multiple steps may be required. Corrective splint use for prolonged period is needed to prevent recurrence of contracture and achieving full correction.

\section{Clinical message}

Deep seated neglected infection in soft tissues in adult may cause a picture of sub clinical ischemic contracture. Prompt diagnosis, suitable treatment and proper positioning of joints and active as well as assisted full range of motion exercises are necessary to prevent and treat ensuing contractures and deformities.

\section{Acknowledgments}

None.

\section{Conflicts of interest}

The authors declare no conflicts of interest.

\section{References}

1. Whitaker AT, Vuillermin C. Lower extremity growth and deformity. Curr Rev in Musculoskeletal Med. 2016;9(4):454-461.

2. Mosca VS. Principles of lower extremity deformity correction in children. Journal of Paediatric Orthopaedics. 2017;37:9-11.

3. Louis S, Charles W. Orthopaedic diagnosis. Apley's system of orthopaedics and fracture. 9th edn, Hodder Arnold. 2010;228

4. Nambi GS, Amisha AK Shah. Additional effect if Iyenger yoga and EMG biofeedback on pain and functional disability in chronic unilateral knee osteoarthritis. International Journal of Yoga. 2013;6(2):123-127.

5. Skalsky AJ, McDonald CM. Prevention and management of limb contractures in neuromuscular diseases. Phys Med Rehabil Clin N Am. 2012;23(3):675-687.

6. Specter SA, Simard CP, Fournier M, et al. Architectural alterations of rat hind limb skeletal muscles immobilized at different lengths. Exp Neurol.1982;76(1):94-110

7. D’Angelo MG, Berti M, Piccinini L, et al. Gait pattern in duchenne muscular dystrophy. Gait Posture. 2009;29(1):36-41.

8. Louis S, Charles W. Orthopaedic diagnosis. apley's system of orthopaedics and fracture. $9^{\text {th }}$ edn, Hodder Arnold. 2010;14.

9. Ranade AS, Belthur MV. Poliomyelitis treatment and management. Symposium on Medscape; 2018. 